International Journal of Applied Mathematics

Volume 34 No. $4 \quad 2021,803-815$

ISSN: 1311-1728 (printed version); ISSN: 1314-8060 (on-line version)

doi: http://dx.doi.org/10.12732/ijam.v34i4.15

\title{
STABILITY ANALYSIS OF CHAOTIC NEW HAMILTONIAN SYSTEM BASED ON HÉNON-HEILES MODEL \\ USING ADAPTIVE CONTROLLED HYBRID PROJECTIVE SYNCHRONIZATION
}

\author{
Ayub Khan ${ }^{1}$, Harindri Chaudhary ${ }^{2} \S$ \\ ${ }^{1}$ Department of Mathematics \\ Jamia Millia Islamia, INDIA \\ ${ }^{2}$ Department of Mathematics \\ Jamia Millia Islamia, INDIA \\ and \\ Department of Mathematics \\ Deshbandhu College (University of Delhi), INDIA
}

\begin{abstract}
This research article deals with a systematic approach to investigate hybrid projective synchronization among identical new chaotic Hamiltonian systems using adaptive control method. First, nonlinear adaptive controllers are designed to estimate the unknown parameters of the given system and also to attain the stability criteria of the error dynamics of the system. Second, the required hybrid projective synchronization in the considered identical systems via adaptive control method is achieved by using Lyapunov stability theory. Additionally, numerical simulations are conducted using MATLAB software to show the efficient performances of the proposed adaptive controller design. Remarkably, both the analytical as well as computational results are in excellent agreement. Moreover, the considered technique has many applications in the field of secure communication and image encryption.
\end{abstract}

AMS Subject Classification: 34K23, 34K35, 37B25, 37N35

Key Words: adaptive control; chaotic system; Lyapunov stability theory; anti-synchronization; MATLAB

Received: March 28, 2021

(C) 2021 Academic Publications

$\S$ Correspondence author 


\section{Introduction}

Undoubtedly, the chaos synchronization and chaos control (CSCC) have been active research areas in applied mathematics for the past three decades. More precisely, CSCC focuses on the behaviour exploration of immensely irregular or disordered nonlinear dynamical systems and plays a significant role in several fields, for instance, secure communication [17], robotics [18], neural networks [1], biomedical engineering [21], ecological models [23], finance models [26], oscillations [4], jerk systems [28], encryption [29], etc. Subsequently, CSCC have sought significant attention in various research fields.

A significant characteristic of chaotic systems, described as "Butterfly Effect" is high sensitivity dependence on initial conditions. This property of chaotic systems was first reported by Lorenz [15] in 1963 while analysing a weather prediction model. Most importantly, Pecora and Caroll [19] first introduced in 1990 the notion of chaos synchronization. In chaos synchronization phenomenon, the state trajectories of two or more chaotic/ hyperchaotic systems are regulated to follow the similar dynamics. In recent years, chaos synchronization of chaotic systems using various control techniques has become a fascinating and engaging area of study for the researchers and scientists. Many significant techniques are introduced and studied to control $[22,2,8]$ and synchronization $[24,12,25,9,10,17,3,7,11]$ of chaos occurring in dynamical systems.

Specifically, Hubler [6] in 1989 firstly introduced adaptive control method (ACM) in chaotic systems. Since then, many researches have been conducted using ACM $[13,8,10,11]$. Keeping the above discussions in view, our primal aim in this paper is to study hybrid projective synchronization (HPS) among identical newly described Hamiltonian chaotic systems [27] based on HénonHeiles model by ACM. Basically, Hénon and Heiles [5] in 1964 first modeled the Hénon-Heiles model which describes the nonlinear motion of a star around a galactic centre with the motion restricted to a plane.

This paper is organized as follows: ection 2 comprises of few essential preliminaries to be used throughout the paper. Section 3 elucidates the basic structured characteristics of the given Hamiltonian chaotic model in detail. Section 4 investigates of the (HPS) method for the given system via ACM. Section 5 consists of the numerical simulations which are displayed graphically using MATLAB. Section 6 concludes the present paper. 


\section{Preliminaries}

The master system and slave system are considered as:

$$
\begin{aligned}
\dot{z}_{m} & =f_{1}\left(z_{m}\right), \\
\dot{z}_{s} & =f_{2}\left(z_{s}\right)+w,
\end{aligned}
$$

where $z_{m}=\left(z_{m 1}, z_{m 2}, \ldots, z_{m n}\right)^{T}, z_{s}=\left(z_{s 1}, z_{s 2}, \ldots, z_{s n}\right)^{T}$ are the state variables of (1) and (2) respectively, $f_{1}, f_{2}: R^{n} \rightarrow R^{n}$ are two nonlinear continuous vector functions and $w=\left(w_{1}, w_{2}, \ldots, w_{n}\right) \in R^{n}$ is the properly designed controller.

We define the hybrid projective synchronization (HPS) error as:

$$
\lim _{t \rightarrow \infty}\|e(t)\|=\lim _{t \rightarrow \infty}\left\|z_{s}(t)-\zeta z_{m}(t)\right\|=0
$$

for some $\zeta=\operatorname{diag}\left(\zeta_{1}, \zeta_{2}, \ldots, \zeta_{n}\right)$ and $\|\cdot\|$ represents vector norm.

Remark 1. For $\zeta_{1}=\zeta_{2}=\ldots=\zeta_{n}=1$, complete synchronization is achieved.

Remark 2. For $\zeta_{1}=\zeta_{2}=\ldots=\zeta_{n}=-1$, anti-synchronization is attained.

Remark 3. If $\zeta_{i}$ 's are not all zeros and $\zeta_{i} \neq \zeta_{j}$ for some $i$ and $j$, then modified projective synchronization is obtained.

\section{System Description}

Proposed by Vaidyanathan et al. [27], the discussed chaotic system can be written as:

$$
\left\{\begin{array}{l}
\dot{z}_{m 1}=z_{m 2} \\
\dot{z}_{m 2}=-z_{m 1}-2 z_{m 1} z_{m 3}+a z_{m 1}^{2} \\
\dot{z}_{m 3}=z_{m 4} \\
\dot{z}_{m 4}=-z_{m 3}-z_{m 1}^{2}+z_{m 3}^{2}+b z_{m 3}^{4}
\end{array}\right.
$$

where $\left(z_{m 1}, z_{m 2}, z_{m 3}, z_{m 4}\right)^{T} \in R^{4}$ is the state vector and $A$ and $B$ are parameters. When $A=1.5$ and $B=-1.9$, the system (4) exhibits chaos. Also, the 
Lyapunov exponents of system (4) are $L E_{1}=0.0015, L E_{2}=0, L E_{3}=0$, $L E_{1}=-0.0015$. In addition, Figure 1(a-f) display the phase diagrams of (4). However, the detailed analytic study and numerical results for the system (4) can be found in [27].

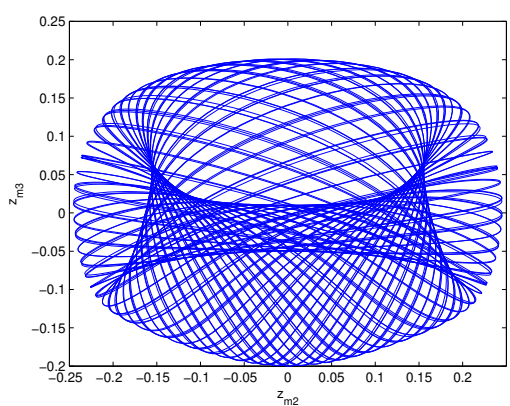

(a)

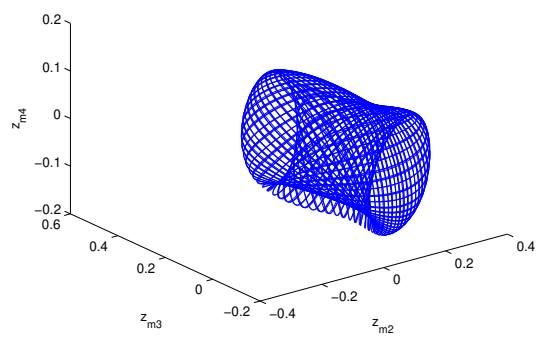

(c)

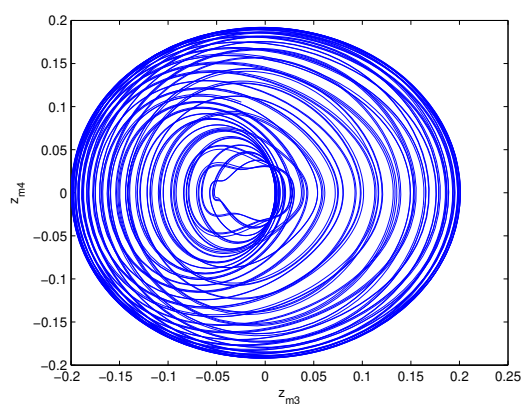

(b)

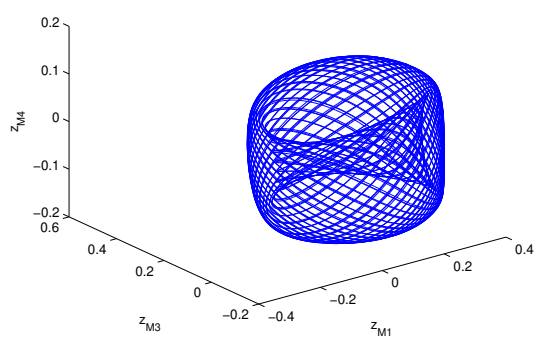

(d)

Figure 1: Phase diagrams of Hamiltonian chaotic system in (a) $z_{m 2}-$ $z_{m 3}$ plane, (b) $z_{m 3}-z_{m 4}$ plane, (c) $z_{m 2}-z_{m 3}-z_{m 4}$ space, (d) $z_{m 1}-$ $z_{m 3}-z_{m 4}$ space

\section{Stability Analysis}

In this section, we discuss HPS scheme to design the laws which estimate parameters with adaptive controllers in such a manner that the state vector $z_{m 1}, z_{m 2}, z_{m 3}$ and $z_{m 4}$ approaches to equilibrium points as $t$ tends to infinity.

The system (4) is selected as the master system and the corresponding slave 
system may be defined as:

$$
\left\{\begin{array}{l}
\dot{z}_{s 1}=z_{s 2}+w_{1} \\
\dot{z}_{s 2}=-z_{s 1}-2 z_{s 1} z_{s 3}+A z_{s 1}^{2}+w_{2} \\
\dot{z}_{s 3}=z_{s 4}+w_{3} \\
\dot{z}_{s 4}=-z_{s 3}-z_{s 1}^{2}+z_{s 3}^{2}+B z_{s 3}^{4}+w_{4}
\end{array}\right.
$$

where $w_{1}, w_{2}, w_{3}$ and $w_{4}$ are adaptive nonlinear controllers to be constructed so that HPS between two identical Hamiltonian chaotic systems will be attained. Also, Figure 2(a-d) show the phase diagrams of the system (5).

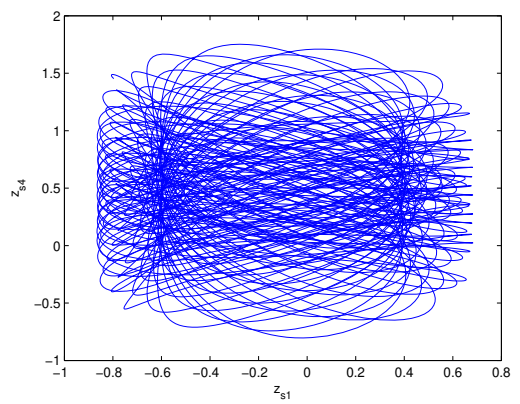

(a)

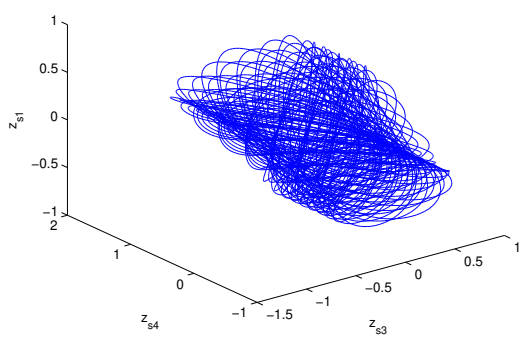

(c)

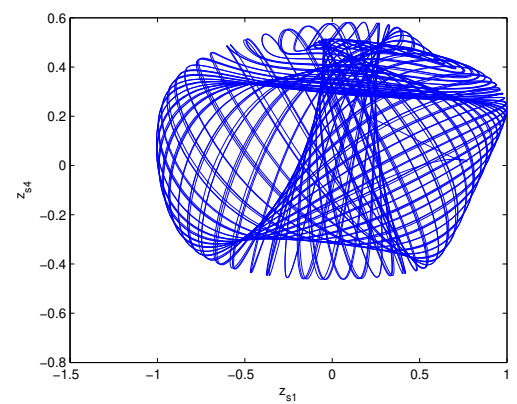

(b)

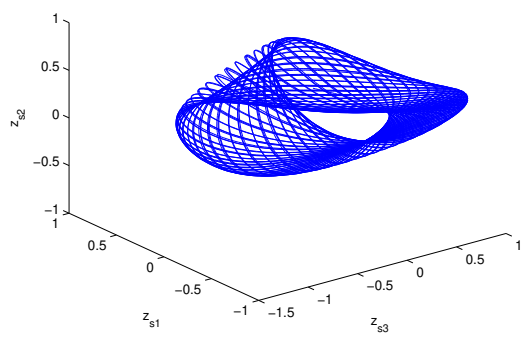

(d)

Figure 2: Phase diagrams of Hamiltonian chaotic system in (a) $z_{s 1}-$ $z_{s 4}$ plane, (b) $z_{s 2}-z_{s 3}$ plane, (c) $z_{s 3}-z_{s 4}-z_{s 1}$ space, (d) $z_{s 3}-z_{s 1}-z_{s 2}$ space 
We formulate the state errors as

$$
\left\{\begin{array}{l}
e_{1}=z_{s 1}-\zeta_{1} z_{m 1} \\
e_{2}=z_{s 2}-\zeta_{2} z_{m 2} \\
e_{3}=z_{s 3}-\zeta_{3} z_{m 3} \\
e_{4}=z_{s 4}-\zeta_{4} z_{m 4}
\end{array}\right.
$$

Our ultimate goal here is to construct controllers $w_{i},(i=1,2,3,4)$ so that the synchronization errors defined in (6) satisfy

$$
\lim _{t \rightarrow \infty} e_{i}(t)=0 \quad \text { for } \quad i=1,2,3,4
$$

The consequent error dynamics turns out to be

$$
\left\{\begin{array}{c}
\dot{e}_{1}=e_{2}+\left(\zeta_{2}-\zeta_{1}\right) z_{m 2}+w_{1} \\
\dot{e}_{2}=-e_{1}+\left(\zeta_{2}-\zeta_{1}\right) z_{m 1}-2\left(z_{s 1} z_{s 3}-\zeta_{2} z_{m 1} z_{m 3}\right) \\
\quad+A\left(z_{s 1}^{2}-\zeta_{2} z_{m 1}^{2}\right)+w_{2} \\
\dot{e}_{3}=e_{4}+\left(\zeta_{4}-\zeta_{3}\right) z_{m 4}+w_{3} \\
\dot{e}_{4}=-e_{3}+\left(\zeta_{4}-\zeta_{3}\right) z_{m 4}-\left(z_{s 1}^{2}-\zeta_{4} z_{m 1}^{2}\right) \\
\quad+\left(z_{s 3}^{2}-\zeta_{4} z_{m 3}^{2}\right)+B\left(z_{s 3}^{4}-\zeta_{4} z_{m 3}^{4}\right)+w_{4}
\end{array}\right.
$$

Next, the adaptive nonlinear controllers are designed by

$$
\left\{\begin{aligned}
w_{1}= & -e_{2}-\left(\zeta_{2}-\zeta_{1}\right) z_{m 2}-L_{1} e_{1} \\
w_{2}= & e_{1}-\left(\zeta_{2}-\zeta_{1}\right) z_{m 1}+2\left(z_{s 1} z_{s 3}-\zeta_{2} z_{m 1} z_{m 3}\right) \\
& \quad-\hat{A}\left(z_{s 1}^{2}-\zeta_{2} z_{m 1}^{2}\right)-L_{2} e_{2} \\
w_{3}= & -e_{4}-\left(\zeta_{4}-\zeta_{3}\right) z_{m 4}-L_{3} e_{3} \\
w_{4}= & e_{3}-\left(\zeta_{4}-\zeta_{3}\right) z_{m 4}+\left(z_{s 1}^{2}-\zeta_{4} z_{m 1}^{2}\right)-\left(z_{s 3}^{2}-\zeta_{4} z_{m 3}^{2}\right) \\
& \quad-\hat{B}\left(z_{s 3}^{4}-\zeta_{4} z_{m 3}^{4}\right)-L_{4} e_{4}
\end{aligned}\right.
$$

where $L_{1}>0, L_{2}>0, L_{3}>0, L_{4}>0$ are gain constants.

On substituting the controllers (8) in error dynamics (7), we get

$$
\left\{\begin{array}{l}
\dot{e}_{1}=-L_{1} e_{1} \\
\dot{e}_{2}=(A-\hat{A})\left(z_{s 1}^{2}-\zeta_{2} z_{m 1}^{2}\right)-L_{2} e_{2} \\
\dot{e}_{3}=-L_{3} e_{3} \\
\dot{e}_{4}=(B-\hat{B})\left(z_{s 3}^{4}-\zeta_{4} z_{m 3}^{4}\right)-L_{4} e_{4}
\end{array}\right.
$$


where $\hat{A}, \hat{B}$ are estimated quantities of unknown parameter $A, B$ respectively.

Defining the parameter estimation error as:

$$
\tilde{A}=A-\hat{A}, \quad \tilde{B}=B-\hat{B} .
$$

Using (10), the error dynamics (9) is written as:

$$
\left\{\begin{array}{l}
\dot{e}_{1}=-L_{1} e_{1} \\
\dot{e}_{2}=\tilde{A}\left(x_{s 1}^{2}-\zeta_{2} x_{m 1}^{2}\right)-L_{2} e_{2} \\
\dot{e}_{3}=-L_{3} e_{3} \\
\dot{e}_{4}=\tilde{B}\left(x_{s 3}^{4}-\zeta_{4} x_{m 3}^{4}\right)-L_{4} e_{4} .
\end{array}\right.
$$

On differentiating parameter estimation error (10), one finds that

$$
\dot{\tilde{A}}=-\dot{\hat{A}}, \quad \dot{\tilde{B}}=-\dot{\hat{B}} .
$$

Constructing the classic Lyapunov function by the rule:

$$
V=\frac{1}{2}\left[e_{1}^{2}+e_{2}^{2}+e_{3}^{2}+e_{4}^{2}+\tilde{A}^{2}+\tilde{B}^{2}\right],
$$

which implying that $V$ is positive definite.

The derivative of Lyapunov function $V$ may be written as:

$$
\dot{V}=e_{1} \dot{e}_{1}+e_{2} \dot{e}_{2}+e_{3} \dot{e}_{3}+e_{4} \dot{e}_{4}-\tilde{A} \dot{\hat{A}}-\tilde{B} \dot{\hat{B}} .
$$

Keeping (14) in mind, we are describing the parameter estimates laws as:

$$
\left\{\begin{array}{l}
\dot{\hat{A}}=\left(z_{s 1}^{2}-\zeta_{2} z_{m 1}^{2}\right) e_{2}+L_{5} \tilde{A} \\
\dot{\hat{B}}=\left(z_{s 3}^{4}-\zeta_{4} z_{m 3}^{4}\right) e_{4}+L_{6} \tilde{B},
\end{array}\right.
$$

where $L_{5}>0$ and $L_{6}>0$ are gain constants.

Theorem 4. The chaotic systems (4)-(5) are asymptotically hybrid projective synchronized for all initial states $\left(z_{m 1}(0), z_{m 2}(0), z_{m 3}(0), z_{m 4}(0)\right) \in R^{4}$ by the designed adaptive controller (8) and the parameter update law (15).

Proof. The Lyapunov function $V$ as considered in (13) is positive definite function. On simplification, Eqns. (11), (14) and (15) reduces to

$$
\dot{V}-L_{1} e_{1}^{2}-L_{2} e_{2}^{2}-L_{3} e_{3}^{2}-L_{4} e_{4}^{2}-L_{5} \tilde{A}^{2}-L_{6} \tilde{B}^{2}<0,
$$


ensuring that $\dot{V}$ is negative definite.

Thus, using LST [20], we conclude that synchronization error $e(t) \rightarrow 0$ exponentially as $t \rightarrow \infty$ for all initial conditions $e(0) \in R^{4}$. This finishes the proof.

\section{Numerical Simulation and Discussion}

This section performs some numerical simulations to illustrate effectively the proposed HPS technique via ACM. $\zeta=\operatorname{diag}\left(\zeta_{1}, \zeta_{2}, \ldots, \zeta_{n}\right)$. The initial states of master (4) and slave systems $(5)$ are $\left(z_{m 1}(0)=0.2, z_{m 2}(0)=0, z_{m 3}(0)=\right.$ $\left.-0.2, z_{m 4}(0)=0\right)$ and $\left(z_{s 1}(0)=0.2, z_{s 2}(0)=0.2, z_{s 3}(0)=-0.2, z_{s 4}(0)=0\right)$ respectively. Also, the scaling matrix $\zeta$ is selected as $\zeta_{1}=4, \zeta_{2}=-3, \zeta_{3}=2$, and $\zeta_{4}=-5$. The control gains are chosen as $L_{i}=6$ for $i=1,2, \ldots, 6$. In addition, simulation results concerning the hybrid projective synchronized trajectories of systems (4) and (5) are shown in Figure 3(a-d). Moreover, Figure 4(a-e) show that the synchronization error $\left(e_{1}, e_{2}, e_{3}, e_{4}\right)=(0.4,0.2,0,0)$ converging to zero as $t$ tending to infinity. In Figure $5(\mathrm{a}-\mathrm{b})$, it is noted that the estimated quantities $(\hat{A}, \hat{B})$ of unknown parameters converging to their original values asymptotical with time. Hence, the proposed HPS synchronization strategy in master and slave systems is achieved computationally.

\section{Conclusion}

In this paper, hybrid projective synchronization in newly designed Hamiltonian chaotic systems has been investigated using ACM. Keeping Lyapunov stability theory in view, adaptive controllers have been described to attain asymptotic stability of the error dynamics of the given system. Further, numerical simulations through MATLAB are presented to validate the efficiency of the proposed methodology. Remarkably, the theoretical results completely agree with the computational results. Such scheme may be utilised to control the nonlinear motion of a star around a galactic centre with motion restricted to a plane. In addition, the proposed strategy may find important applications in the areas of image encryption and secure communication. 


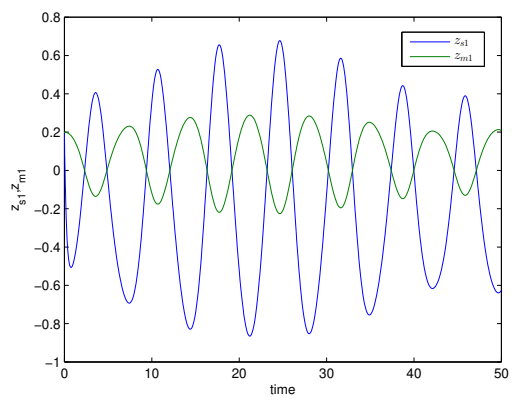

(a)

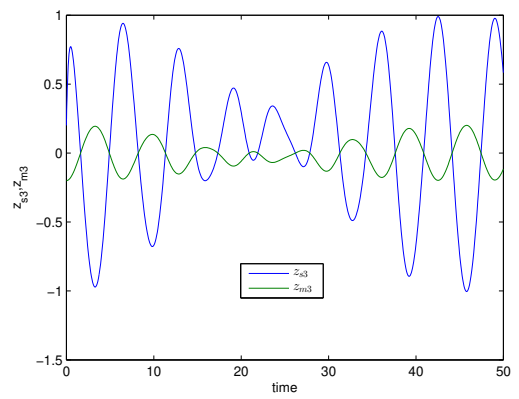

(c)

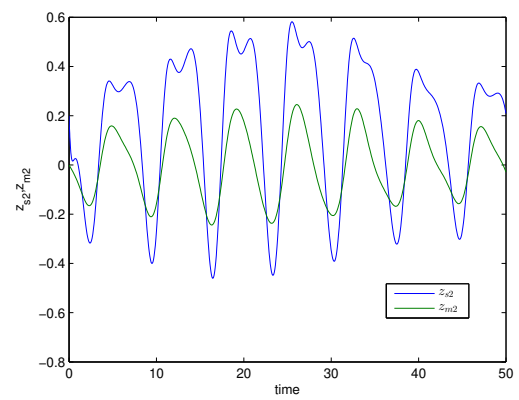

(b)

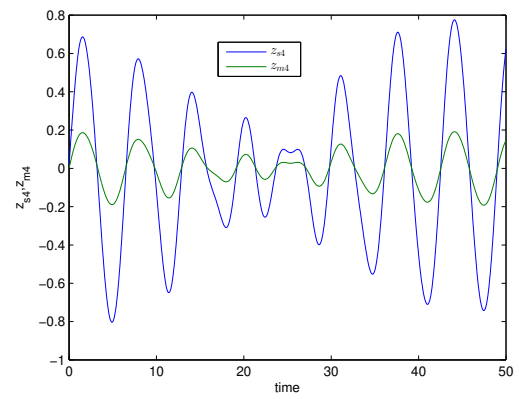

(d)

Figure 3: Hybrid projective synchronization of Hamiltonian chaotic systems (a) between $x_{m 1}(t)-x_{s 1}(t)$, (b) between $x_{m 2}(t)-x_{s 2}(t)$, (c) between $x_{m 3}(t)-x_{s 3}(t),(\mathrm{d})$ between $x_{m 4}(t)-x_{s 4}(t)$

\section{References}

[1] K. Bouallegue, A new class of neural networks and its applications, Neurocomputing, 249 (2017), 28-47.

[2] M. Chen, Z. Han, Controlling and synchronizing chaotic genesio system via nonlinear feedback control, Chaos, Solitons \& Fractals, 17, No 4 (2003), 709-716.

[3] Z. Ding and Y. Shen, Projective synchronization of nonidentical fractionalorder neural networks based on sliding mode controller, Neural Networks, 76 (2016), 97-105. 


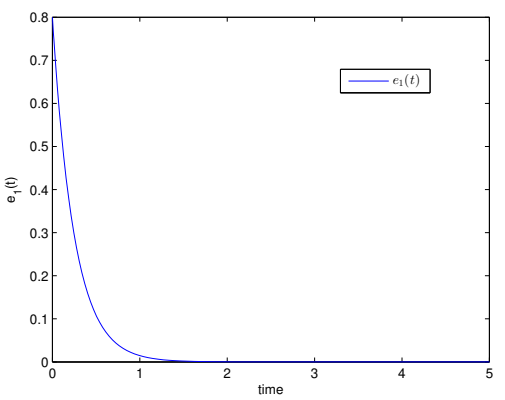

(a)

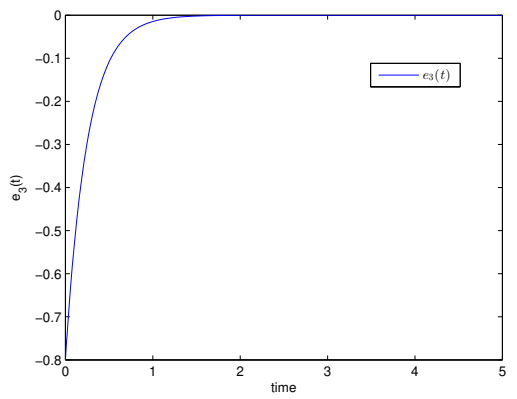

(c)

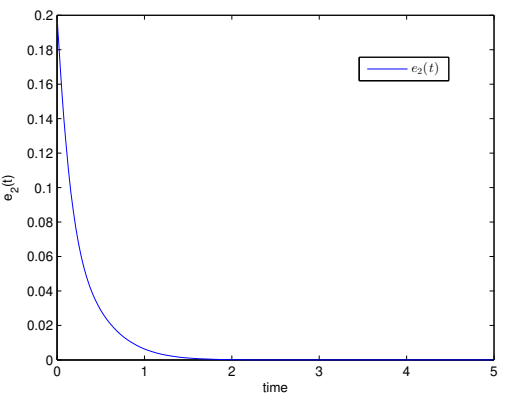

(b)

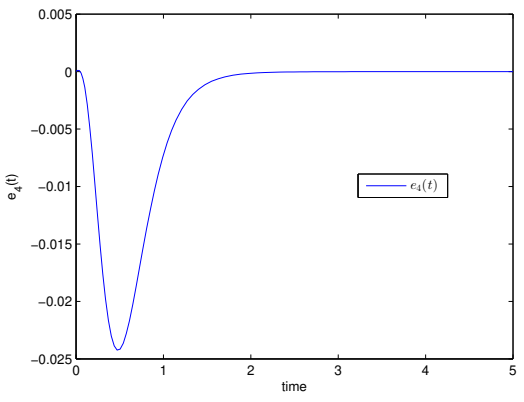

(d)

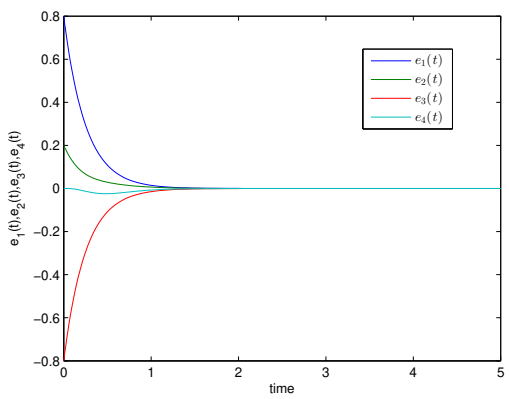

(e)

Figure 4: Dynamics in synchronization error states (a) $\left(t, e_{1}(t)\right)$, (b) $\left(t, e_{2}(t)\right),(\mathrm{c})\left(t, e_{3}(t)\right),(\mathrm{d})\left(t, e_{4}(t)\right),(\mathrm{e})\left(t, e_{1}(t), e_{2}(t), e_{3}(t), e_{4}(t)\right)$

[4] D. Ghosh, A. Mukherjee, N. R. Das, B. N. Biswas, Generation \& control of chaos in a single loop optoelectronic oscillator, Optik, 165 (2018), 275-287. 


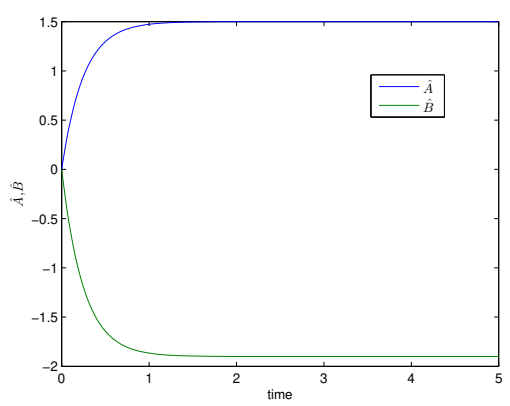

Figure 5: Time history of parameter estimates of Hamiltonian chaotic system

[5] M. Henon, C. Heiles, The applicability of the third integral of motion: some numerical experiments, The Astronomical J., 69 (1964), 73.

[6] A. W. Hubler, Adaptive control of chaotic system, Helv Phys. Acta, 62 (1989), 343-346.

[7] L. S. Jahanzaib, P. Trikha, H. Chaudhary, S. M. Haider et al., Compound synchronization using disturbance observer based adaptive sliding mode control technique, J. Math. Comput. Sci., 10, No 5 (2020), 1463-1480.

[8] A. Khan, H. Chaudhary, Adaptive control and hybrid projective combination synchronization of chaos generated by generalized Lotka-Volterra biological systems, Emer. Trends Infor. Tech., Bloomsbury India (2019), 174-184.

[9] A. Khan, H. Chaudhary, Hybrid projective combination-combination synchronization in non-identical hyperchaotic systems using adaptive control, Arabian J. Math., 9, No 3 (2020), 1-15.

[10] T. Khan, H. Chaudhary, Estimation and identifiability of parameters for generalized Lotka-Volterra biological systems using adaptive controlled combination difference anti-synchronization, Differ. Equ. Dyn. Syst., 28 (2020), 515-526.

[11] S. Kumar, A. E. Matouk, H. Chaudhary, S. Kant, Control and synchronization of fractional-order chaotic satellite systems using feedback and adaptive control techniques, Int. J. Adapt. Control Signal Process., 35, No 4 (2020), 484-497. 
[12] G.-H. Li, S.-P. Zhou, Anti-synchronization in chaotic systems, Chaos, Solitons \& Fractals, 32, No 2 (2007), 516-520.

[13] S.-Y. Li, C.-H. Yang, C.-T. Lin, L.-W. Ko, T.-T. Chiu, Adaptive synchronization of chaotic systems with unknown parameters via new backstepping strategy, Nonlinear Dynamics, 70, No 3 (2012), 2129-2143.

[14] Z. Li, D. Xu, A secure communication scheme using projective chaos synchronization, Chaos, Solitons \& Fractals, 22, No 2 (2004), 477-481.

[15] E. N. Lorenz, Deterministic nonperiodic flow, J. Atmospheric Sci., 20, No 2 (1963), 130-141.

[16] J. Ma, L. Mi, P. Zhou, Y. Xu, T. Hayat, Phase synchronization between two neurons induced by coupling of electromagnetic field, Applied Math. Comput., 307 (2017), 321-328.

[17] B. Naderi, H. Kheiri, A. Heydari, Secure communication based on synchronization of three chaotic systems, Int. J. Nonlinear Sci., 27, No 1 (2019), 53-64.

[18] B. K. Patle, D. R. K. Parhi, A. Jagadeesh, S. K. Kashyap, Matrix-binary codes based genetic algorithm for path planning of mobile robot, Computers \& Electrical Eng., 67 (2018), 708-728.

[19] L. M. Pecora, T. L. Carroll, Synchronization in chaotic systems, Phys. Rev. Lett., 64, No 8 (1990), 821-824.

[20] L. Perko, Differential Equations and Dynamical Systems, Vol 7, Springer Science \& Business Media (2013).

[21] A. Provata, P. Katsaloulis, D. A. Verganelakis, Dynamics of chaotic maps for modelling the multifractal spectrum of human brain diffusion tensor images, Chaos, Solitons \& Fractals, 45, No 2 (2012), 174-180.

[22] S. Rasappan, S. Vaidyanathan, Synchronization of hyperchaotic liu system via backstepping control with recursive feedback, In: Int. Conf. on Ecofriendly Comput. Commu. Syst., 212-221, Springer, 2012.

[23] B. Sahoo, S. Poria, The chaos and control of a food chain model supplying additional food to top-predator, Chaos, Solitons \& Fractals, 58 (2014), $52-64$. 
[24] A. K. Singh, V. K. Yadav, S. Das, Synchronization between fractional order complex chaotic systems, Int. J. Dyn. Control, 5, No 3 (2017), 756-770.

[25] K. S. Sudheer, M. Sabir, Hybrid synchronization of hyperchaotic lu system, Pramana, 73, No 4:781, 2009.

[26] X.-J. Tong, M. Zhang, Z. Wang, Y. Liu, J. Ma, An image encryption scheme based on a new hyperchaotic Finance system, Optik, 126, No 20 (2015), 2445-2452.

[27] S. Vaidyanathan, A. Sambas, S. Zhang, M. A. Mohamed, M. Mamat, A new hamiltonian chaotic system with coexisting orbits and its dynamical analysis, Int. J. Eng. Tech., 7, No 4 (2018), 2430-2436.

[28] X. Wang, S. Vaidyanathan, C. Volos, V.-T. Pham, T. Kapitaniak, Dynamics, circuit realization, control and synchronization of a hyperchaotic hyperjerk system with coexisting attractors, Nonlinear Dynamics, 89, No 3 (2017), 1673-1687.

[29] G.-C. Wu, D. Baleanu, Z.-X. Lin, Image encryption technique based on fractional chaotic time series, J. Vibr. Control, 22, No 8 (2016), 20922099. 
